ISSN 0103-5150

Fisioter. Mov., Curitiba, v. 25, n. 1, p. 105-115, jan./mar. 2012 Licenciado sob uma Licença Creative Commons

\title{
Teste do degrau e teste da cadeira: comportamento das respostas metábolo-ventilatórias e cardiovasculares na DPOC
}

\author{
Step test and sit-to-stand test: behavior of metabolic, ventilatory \\ and cardiovascular responses in patients with COPD
}

\author{
Bruna Varanda Pessoa ${ }^{[a]}$, Mauricio Jamami ${ }^{[b]}$, Renata Pedrolongo Basso ${ }^{[\mathrm{c}]}$, Eloisa Maria Gatti \\ Regueiro $^{[\mathrm{d}]}$, Valéria Amorim Pires Di Lorenzo ${ }^{[\mathrm{e}]}$, Dirceu Costa ${ }^{[\mathrm{f}]}$
}

[a] Doutoranda do Programa de Pós-Graduação em Fisioterapia da Universidade Federal de São Carlos (UFSCar), São Carlos, SP Brasil, e-mail: brunavpessoa@hotmail.com

[b] Professor Doutor adjunto do curso de Graduação em Fisioterapia e Programa de Pós-Graduação em Fisioterapia da Universidade Federal de São Carlos (UFSCar), São Carlos, SP - Brasil, e-mail: jamami@ufscar.br

[c] Doutoranda do Programa de Pós-Graduação em Fisioterapia da Universidade Federal de São Carlos (UFSCar), São Carlos, SP Brasil, e-mail: renata.fisio@gmail.com

[d] Doutoranda do Programa de Pós-Graduação em Fisioterapia da Universidade Federal de São Carlos (UFSCar), São Carlos, SP Brasil, e-mail: eloregueiro@yahoo.com.br

[e] Professora Doutora adjunta do curso de Graduação em Fisioterapia e Programa de Pós-Graduação em Fisioterapia da Universidade Federal de São Carlos (UFSCar), São Carlos, SP - Brasil, e-mail: vallorenzo@ufscar.br

[f] Professor Doutor do curso de Pós-Graduação em Fisioterapia da Universidade Nove de Julho (UNINOVE), São Paulo, SP Brasil, e-mail: dcosta@uninove.br

\section{Resumo}

Objetivos: Verificar as respostas metabólicas, ventilatórias, cardiovasculares e de percepção de esforço entre o isotime de dois minutos do teste do degrau (TD2) e o teste do degrau de seis minutos (TD6) com o teste de sentar-se e levantar-se da cadeira de dois minutos (TSL) nos indivíduos com DPOC. Materiais e métodos: Foram avaliados 11 homens com DPOC ( $71 \pm 8$ anos, $\mathrm{VEF}_{1}=46,1 \pm 15,2 \%$ previsto), clinicamente estáveis, por meio do TD6 e TSL associados à análise de gases e à coleta de lactato sanguíneo, realizadas em dias diferentes e não consecutivos. No TD2 e TD6, os indivíduos foram instruídos a subir e descer um degrau de $20 \mathrm{~cm}$ de altura o mais rápido possível. 0 mesmo foi recomendado no TSL, que foi realizado em uma cadeira de $46 \mathrm{~cm}$ de altura. Resultados: Na análise intertestes não se observou diferença significativa nas variáveis metábolo-ventilatórias, cardiovasculares e $\Delta$ dispneia no pico de ambos os testes, bem como 
no TD2. Quanto ao $\Delta$ frequência cardíaca e ao $\Delta$ fadiga nos membros inferiores, constataram-se valores significantemente maiores para o TSL comparado ao TD2; e correlações positivas entre o consumo de oxigênio, $\Delta$ frequência cardíaca e os desempenhos no TD2 e TD6, entre os desempenhos no TD6 e TSL, e no TD2 com TSL. Conclusão: Os testes realizados apresentaram respostas metábolo-ventilatórias, cardiovasculares e dispneia similares; e o TD2 mostrou-se uma alternativa para avaliar as limitações funcionais dos indivíduos com DPOC de obstrução grave, proporcionando menor estresse cardiovascular e fadiga muscular se comparado ao TSL, pelas exigências metabólicas periféricas e ajustes posturais.

Palavras-chave: Doença pulmonar obstrutiva crônica. Teste de esforço. Tolerância ao exercício. Consumo de oxigênio. Fadiga muscular.

\section{Abstract}

objectives: Verify the metabolic, ventilatory, cardiovascular and perceived effort responses between the isotime two minute step test (2MST) and six minute step test (6MST) with two minute sit-to-stand test (STST) in individuals with COPD. Materials and methods: 11 men with COPD $(71 \pm 8$ years, FEV1 $=46.1 \pm 15.2 \%$ predicted), clinically stable, were evaluation by STST and 6MST associated gas analysis and blood lactate in days which were not consecutive and not coincidental. In the 6MST and 2MST, the subjects were instructed ascend-todescend a step $20 \mathrm{~cm}$ in height as quickly as possible. It was recommended that in the STST was performed in a chair $46 \mathrm{~cm}$ high. Results: In the inter-tests analysis, there was no significant difference in the metabolic, ventilatory, cardiovascular variables and delta of variation ( $\Delta$ ) dyspnea at the peak of both tests, and 2MST. The $\Delta$ heart frequency and $\triangle$ fatigue in the lower limbs were significantly higher compared to STST for 2MST. We observed moderate and strong positive correlations between oxygen consumption, $\Delta$ heart frequency and performances in 2MST and 6MST, between performances in 6MST and STST, and 2MST with STST. Conclusion: The tests showed metabolic, ventilatory, cardiovascular and dyspnoea responses similar; and 2MST, can be alternative to assess the functional limitations of individuals with COPD severe obstruction, providing a lower cardiovascular stress and muscle fatigue compared to the STST, the peripheral metabolic requirements and postural adjustments.

Keywords: Chronic obstructive pulmonary disease. Exercise test. Exercise tolerance. Oxygen consumption. Muscle fatigue.

\section{Introdução}

A intolerância ao exercício nos indivíduos com doença pulmonar obstrutiva crônica (DPOC) tem importante implicação na qualidade de vida, no número de hospitalizações e na sobrevida (1). Dessa maneira, os testes físicos funcionais têm sido considerados componentes essenciais na rotina clínica de avaliação da capacidade de exercício (1).

Dentre eles, cita-se o teste cardiopulmonar (TCP), considerado o ideal para avaliar a tolerância máxima ao exercício e determinar a etiologia da limitação ao exercício, fornecendo informações mais específicas sobre a capacidade funcional e a adaptação fisiológica diante do esforço físico desses indivíduos (1-4); mas, pelo alto custo e complexidade do equipamento, ainda é pouco utilizado fora dos grandes centros de pesquisas e está longe de ser efetivo, principalmente para a realidade do serviço público de saúde brasileiro $(2,4,5)$.

Como formas alternativas de avaliação aos testes máximos, destacam-se os testes funcionais submáximos $(1,6-8)$, por mostraram-se opções de fácil realização, baixa complexidade, além de não requererem equipamentos de alto custo, sendo de fácil aplicação na prática clínica $(1,3,5)$; com destaque para o teste de caminhada de seis minutos (TC6) (9), teste do degrau de seis minutos (TD6) (7) e o teste da cadeira (8), utilizados na prática clínica para avaliação funcional da tolerância ao exercício, prescrição de treinamento e para avaliar as mudanças observadas após um programa de treinamento físico.

0 TC6 apresenta boa reprodutibilidade, confiabilidade e baixo custo $(3,6,9)$, além de ser um preditor de morbidade e mortalidade $(10,11)$, e refletir a 
capacidade de exercício de indivíduos com DPOC ao realizar as atividades físicas da vida diária (12-14). Entretanto, o espaço físico necessário para sua execução muitas vezes é um fator limitante à sua utilização $(7,9)$.

Por outro lado, o TD6 e o teste da cadeira requerem mínimo espaço físico e têm se mostrado úteis clinicamente na estimativa da tolerância ao exercício, uma vez que impõem alta demanda metabólica quando comparados ao TC6, sendo considerados boas alternativas para a avaliação da capacidade de exercício e a limitação ao esforço desses indivíduos $(4,7,8,14,15)$.

Schnaider e Karsten (15) verificaram que o TD6 pode substituir o TC6 em ambiente hospitalar na avaliação da tolerância ao exercício em indivíduos DPOC exacerbados, e Montes de Oca et al. (16) concluíram que o TD6 foi melhor que o TC6 para determinar a capacidade funcional máxima de indivíduos com DPOC, porém não avaliaram as variáveis ergoespirométricas durante o teste. E Dal Corso et al. (7) concluíram que o TD6 fornece estimativas confiáveis e reprodutíveis da capacidade de exercício e da dessaturação da oxiemoglobina em pacientes com doença pulmonar intersticial. Além disso, Ozalevli et al. (8) encontraram que o teste da cadeira comparado ao TC6 é hemodinamicamente menos estressante e mais sensível para avaliar a capacidade funcional de indivíduos com DPOC, sendo uma alternativa de substituição.

Entretanto, há poucos estudos na literatura sobre a padronização e o tempo de execução do teste do degrau e da cadeira, bem como sobre as respostas metábolo-ventilatórias e cardiovasculares obtidas durante a realização de tais estudos $(7,8)$, sugerindo que um teste do degrau com o tempo de dois minutos forneceria uma estimativa confiável da capacidade de exercício dos indivíduos com DPOC de obstrução grave, além de serem suficientes para provocarem alterações metabólicas, ventilatórias e cardiovasculares; justificando a realização deste estudo.

Sendo assim, o objetivo deste estudo foi verificar as respostas metabólicas, ventilatórias, cardiovasculares e de percepção de esforço entre o isotime de dois minutos do teste do degrau (TD2) e o teste do degrau de seis minutos (TD6) com o teste de sentar-se e levantar-se da cadeira em dois minutos (TSL) nos indivíduos com DPOC. Além disso, objetivou-se relacionar essas variáveis e a capacidade de exercício entre os testes.

\section{Materiais e métodos}

\section{Participantes}

Foram avaliados inicialmente 16 indivíduos do sexo masculino, na faixa etária de 58 aos 81 anos de idade, apresentando DPOC de obstrução moderada a muito grave (relação volume expiratório forçado no primeiro segundo $\left[\mathrm{VEF}_{1}\right]$ pela capacidade vital

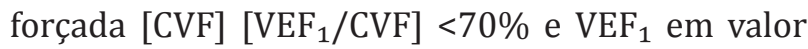
percentual do previsto $\left(\mathrm{VEF}_{1} \%\right.$ prev) $<80$ (estágios II, III e IV) (17).

De acordo com os critérios de inclusão do estudo, consideraram-se aptos a participar os indivíduos do sexo masculino, com diagnóstico clínico e espirométrico de DPOC, confirmado pela espirometria pré e pós-BD - realizada sob a supervisão de um pneumologista - e classificado como obstrução moderada a muito grave (17); os que não apresentaram doenças cardíacas, reumáticas e ortopédicas associadas que os impedissem de realizar o protocolo proposto; os clinicamente estáveis, sem história de infecções ou exacerbação dos sintomas respiratórios ou mudança de medicamentos nos três meses anteriores ao estudo; além dos que não realizaram programa de treinamento físico regular por um período de seis meses anteriores ao estudo; não tabagistas ou ex-tabagistas; e que apresentaram o escore do questionário mini-exame do estado mental (18) para avaliação da capacidade cognitiva $>25$.

Foram excluídos cinco indivíduos, um por apresentar DPOC de obstrução leve e problemas ortopédicos; um com obstrução moderada, que desistiu por problemas pessoais; e três indivíduos com obstrução grave, sendo um em razão da intolerância ao uso da máscara de análise de gases e dois por apresentarem problemas ortopédicos. A amostra final foi composta por 11 indivíduos com DPOC.

Dos 11 indivíduos que completaram o estudo, quatro apresentavam DPOC de obstrução moderada (estádio II); cinco, obstrução grave (estádio III); e dois, muito grave (estádio IV) (17). Todos faziam uso de broncodilatador (BD), sendo que dez associavam-no ao uso de corticosteroide inalatório (dose média de $400 \mathrm{mcg}$ ) e um, ao uso de corticosteroide oral (dose média de $20 \mathrm{mg}$ ) sob prescrição médica por pelo menos seis meses anteriores ao estudo. Desses indivíduos, somente três eram reagentes ao $\mathrm{BD}$, sendo que nove apresentavam distúrbio ventilatório obstrutivo (DVO) com redução da CVF por hiperinsuflação, um 
apresentava DVO apenas com redução da CVF e um, distúrbio ventilatório misto (19).

Todos assinaram o Termo de Consentimento Livre e Esclarecido mediante orientações sobre o protocolo proposto em atendimento à Resolução n. 196/96 do Conselho Nacional de Saúde. Este estudo foi aprovado pelo Comitê de Ética da instituição, Parecer n. 074/2007.

\section{Procedimento experimental}

Os indivíduos foram encaminhados à Unidade de Fisioterapia Respiratória da instituição e submetidos a uma avaliação física geral e específica do sistema respiratório; além do teste espirométrico pré e pós-BD sob responsabilidade de um pneumologista de acordo com as normas da American Thoracic Society/European Respiratory Society (ATS/ERS) (20), sendo obtidas as medidas de capacidade vital lenta, $\mathrm{CVF}, \mathrm{VEF}_{1}$, a relação $\mathrm{VEF}_{1} / \mathrm{CVF}$ e a ventilação voluntária máxima.

\section{Testes de avaliação funcional}

0 TD6 e o TSL associados à análise de gases foram realizados em dias não consecutivos, com intervalo de no mínimo 24 horas $(16,21)$, randomizados por sorteio, e cada teste foi realizado duas vezes com um intervalo mínimo de 30 minutos entre eles, para eliminar o efeito do aprendizado, com o melhor desempenho utilizado para análise, sendo todas as avaliações realizadas no período da tarde e em uma sala climatizada.

Os princípios gerais do TD6 e TSL foram baseados nas recomendações estabelecidas pela ATS (9) para o TC6, sendo os indivíduos incentivados com frases preestabelecidas, em intervalos padronizados de um minuto.

\section{Teste do degrau de seis minutos}

O TD6 foi realizado em um degrau de $20 \mathrm{~cm}$ de altura, com piso de borracha antiderrapante (7). Visando à melhor funcionalidade, o teste seguiu os mesmos princípios da ATS (9) para o TC6, sendo os indivíduos instruídos a subir e descer o degrau o mais rápido possível durante seis minutos, intercalando os
MMII, sem o apoio dos membros superiores (MMSS), e a diminuir a cadência ou a interromper o teste em caso de fadiga intensa (7) ou atingir a FC submáxima (85\% da FC máxima), sendo a cadência de execução livre de acordo com Dal Corso et al. (7). Foram registradas as variáveis no TD2 para efeito de comparação com as variáveis do TSL.

Teste de sentar-se e levantar-se da cadeira em dois minutos

0 TSL foi adaptado e modificado segundo metodologia descrita por Ozalevli et al. (8), e tem sido recomendado como alternativa prática para mensurar indiretamente a força dos MMII, sendo considerado um preditor de gravidade. Foi utilizada uma cadeira com encosto, sem apoio para os MMSS e altura de $46 \mathrm{~cm}$ (8). 0 teste iniciou-se com o indivíduo sentado na cadeira, com as costas apoiadas no encosto e os pés apoiados no chão. Tal indivíduo foi orientado a sentar completamente na cadeira, levantar-se estendendo totalmente os joelhos, sem realizar compensações posturais, mantendo os MMSS cruzados à frente do tórax. Todos os indivíduos deveriam repetir o procedimento o mais rápido possível (8), tantas vezes quanto possível, em um período de dois minutos, sendo a cadência de execução do teste livre.

\section{Medidas de monitorização}

Foram registradas no repouso (basal), no segundo, quarto e sexto minutos do TD6, e no repouso, primeiro e segundo minutos no TSL, além do primeiro, terceiro e sexto minutos da recuperação em ambos os testes, a saturação periférica de oxigênio $\left(\mathrm{SpO}_{2}\right)$ por meio de um oxímetro de pulso portátil (Nonin ${ }^{\circledR}$, modelo 2500, Minneapolis, Mn, USA), a frequência cardíaca $(\mathrm{FC})$ por meio de um frequencímetro de pulso (Polar Vantage NVTM ${ }^{\circledR}$, modelo 1901001, Kempele, Oulu, Finlândia), e os sintomas de dispneia e fadiga muscular de MMII, sendo as respostas classificadas pela escala de Borg modificada CR10 (22). As medidas da pressão arterial, mensuradas com o indivíduo na posição sentada e com um esfigmomanômetro de coluna de mercúrio (Oxigel ${ }^{\circledR}$, São Paulo, SP, Brasil), foram verificadas no repouso, imediatamente após os testes e no primeiro, terceiro e sexto minutos da recuperação. 
A reserva de $\mathrm{FC}(\mathrm{RFC})$ foi obtida de forma indireta pela fórmula: [(FCmax-FC obtida no teste)/ FCmax]x100 (4), sendo a FCmax = 220 - idade.

\section{Análise dos gases}

A coleta dos gases respiratórios foi realizada durante o segundo TD6 e TSL, por meio de um analisador de gases modelo $\mathrm{VO}_{2000}$ da MedGraphics ${ }^{\circledR}$ (St Paul, MN, USA), por meio de um pneumotacógrafo de baixo fluxo bidirecional acoplado a uma máscara. Esse sistema mede microamostras dos gases expirados pelo método de coleta da média de três respirações. Os dados produzidos foram digitalizados para o computador, em tempo real, via porta serial, e plotados em gráficos para as seguintes variáveis selecionadas: - $\mathrm{V}_{\mathrm{E}}$ : volume de ar expirado em litros por minuto, expresso em body temperature pressure saturated (BTPS); - $\mathrm{VO}_{2}$ : volume de oxigênio consumido em litros por minuto, expresso em standard temperature and pressure dry (STPD); e - $\mathrm{VCO}_{2}$ : produção de gás carbônico consumido em litros por minuto, expresso em STPD. Para o cálculo da $\mathrm{RV}_{\mathrm{E}}$ e $\mathrm{RVO}_{2}$ foram utilizadas as fórmulas citadas por Neder e Nery (4), além disso foi calculada a razão $\mathrm{V}_{\mathrm{E}} / \mathrm{VVM} \mathrm{e} \mathrm{VO}_{2} / \mathrm{VO}_{2}$ max.

Antes dos testes os indivíduos permaneceram sentados para colocação da máscara facial e adaptação ao sistema. Após a estabilização das variáveis metabólicas e ventilatórias foram realizadas as coletas durante três minutos na postura ortostática (repouso) e durante todo o teste até o período de recuperação.

\section{Coleta e análise do lactato sanguíneo}

Foram coletadas amostras de sangue $(25 \mu \mathrm{L})$ do lóbulo da orelha por meio de capilares de vidro heparinizados e calibrados para posterior determinação das concentrações de lactato no início (repouso) e ao fim (pico) do TD6 e TSL. 0 sangue foi depositado em Ependorfs, contendo $50 \mu \mathrm{l}$ de fluoreto de sódio a $1 \%$, o que contribuiu para evitar a continuidade da glicólise e provocar hemólise. Após as coletas do sangue, as amostras foram estocadas em freezer e posteriormente foram levadas ao laboratório para serem analisadas por meio do lactímetro YSI (Yellow Springs Inc., modelo 1500 Sport USA), e a determinação do lactato sanguíneo foi realizada pelo método eletroenzimático.
Análise estatística

Os resultados do estudo foram analisados pelo pacote estatístico Statistical Package for Social Sciences para Windows, versão 16.0 (SPSS Inc, Chicago, IL). A distribuição dos dados foi verificada pelo teste de normalidade de Shapiro-Wilk. Os dados foram expressos em médias \pm desvios-padrões. Para a análise intertestes foi utilizado o teste $t$ de Student pareado para todas as variáveis analisadas.

0 cálculo do poder amostral foi realizado pelo programa GraphPad StatMate 2.0 para Windows, baseado em um estudo piloto no desvio-padrão e na diferença das médias para todas as variáveis analisadas, correspondendo a um poder acima de $80 \%$. 0 nível de significância adotado foi de $5 \%$.

\section{Resultados}

As variáveis antropométricas e espirométricas estão apresentadas na Tabela 1. 0 tratamento medicamentoso manteve-se constante durante o período do estudo. Todos os indivíduos completaram com sucesso ambos os testes.

Tabela 1 - Características antropométricas e espirométricas dos grupos estudados

\begin{tabular}{|c|c|c|}
\hline \multirow[t]{2}{*}{ Variáveis } & \multicolumn{2}{|c|}{ Valores $(n=11 H)$} \\
\hline & Média \pm DP & Valor min-max \\
\hline Idade (anos) & $70,5 \pm 7,7$ & $58,0-81,0$ \\
\hline Peso (kg) & $69,8 \pm 9,4$ & $48,0-84,0$ \\
\hline Altura (cm) & $165,6 \pm 4,9$ & $155,5-172,0$ \\
\hline IMC $\left(\mathrm{kg} / \mathrm{m}^{2}\right)$ & $25,4 \pm 2,9$ & $19,9-29,3$ \\
\hline $\operatorname{VEF}_{1}(\%$ prev) & $46,1 \pm 15,2$ & $23,2-66,3$ \\
\hline CVF (\% prev) & $76,5 \pm 15,1$ & $59,6-103,2$ \\
\hline $\operatorname{VEF}_{1} /$ CVF (\%) & $62,4 \pm 11,9$ & $41,7-66,5$ \\
\hline VVM (\% prev) & $51,4 \pm 17,2$ & $18,0-71,0$ \\
\hline
\end{tabular}

Legenda: Legenda: $\mathrm{n}=$ número de indivíduos; $\mathrm{H}=$ homens; IMC = índice de massa corporal; VEF $_{1}=$ volume expiratório forçado no primeiro segundo; CVF = capacidade vital forçada; $V_{1} F_{1} / C V F=$ razão $V_{1} F_{1} / C V F ; ~ V V M=$ ventilação voluntária máxima.

Fonte: Dados da pesquisa. 
$\mathrm{Na}$ análise intertestes, comparando-se as situações: basal do TD6 vs. basal do TSL; pico do TD6 vs. pico do TSL; e TD2 vs. pico do TSL, não se observou diferença significativa $(\mathrm{p}>0,05)$ nas variáveis metabólicas [consumo de oxigênio $\left(\mathrm{VO}_{2}\right)$, produção de gás carbônico $\left(\mathrm{VCO}_{2}\right)$, reserva metabólica $\left(\mathrm{RVO}_{2}\right)$, quociente respiratório $(\mathrm{QR})$, demanda metabólica $\left(\mathrm{VO}_{2} / \mathrm{VO}_{2} \mathrm{max}\right)$ e lactato sanguíneo] (Tabela 2); ventilatórias [ventilação pulmonar $\left(\mathrm{V}_{\mathrm{E}}\right)$, reserva ventilatória $\left(\mathrm{RV}_{\mathrm{E}}\right)$, equivalente ventilatório para o oxigênio $\left(\mathrm{V}_{\mathrm{E}} / \mathrm{VO}_{2}\right)$, equivalente ventilatório para o gás carbônico $\left(\mathrm{V}_{\mathrm{E}} / \mathrm{VCO}_{2}\right)$, demanda ventilatória $\left(\mathrm{V}_{\mathrm{E}} / \mathrm{VVM}\right)$, $\Delta \mathrm{SpO}_{2}$ e $\Delta \mathrm{FR}$ ] (Tabelas 2 e 3); cardiovasculares [pulso de oxigênio $\left(\mathrm{VO}_{2} / \mathrm{FC}\right), \mathrm{RFC}, \Delta \mathrm{PAS}$ e $\Delta \mathrm{PAD}$ ] (Tabelas 2 e 3) e $\Delta$ sensação de dispneia (Tabela 3).

Quanto ao $\Delta \mathrm{FC}$ e ao $\Delta$ fadiga nos MMII constataram-se valores significantemente maiores $(\mathrm{p}<0,05)$ para o pico do TSL comparado ao TD2, conforme demonstrado na Tabela 3.

Constatou-se dessaturação em todos os testes submáximos realizados. Da amostra total, seis indivíduos apresentaram dessaturação de oxigênio no TD6 e cinco, no TSL. Não foi administrada a suplementação de oxigênio durante os testes funcionais submáximos conforme descrito na metodologia (7).

Tabela 2 - Variáveis metabólicas, ventilatórias e cardiovasculares nas situações basal e pico dos testes, e isotime de dois minutos do teste do degrau dos indivíduos com DPOC

\begin{tabular}{|c|c|c|c|c|c|}
\hline \multirow[t]{2}{*}{ Variáveis } & \multicolumn{3}{|c|}{ TD6 } & \multicolumn{2}{|c|}{ TSL } \\
\hline & Basal & Pico & Isotime (TD2) & Basal & Pico \\
\hline \multicolumn{6}{|l|}{ Metabólicas } \\
\hline $\mathrm{VO}_{2}(\mathrm{~L} / \mathrm{min})$ & $0,2 \pm 0,1$ & $0,9 \pm 0,3$ & $0,7 \pm 0,3$ & $0,2 \pm 0,1$ & $0,8 \pm 0,3$ \\
\hline $\mathrm{VCO}_{2}(\mathrm{~L} / \mathrm{min})$ & $0,2 \pm 0,1$ & $1,0 \pm 0,4$ & $0,7 \pm 0,3$ & $0,3 \pm 0,1$ & $0,9 \pm 0,3$ \\
\hline $\mathrm{RVO}_{2}(\%)$ & $86,0 \pm 9,1$ & $39,4 \pm 13,9$ & $54,0 \pm 15,1$ & $83,7 \pm 4,3$ & $48,2 \pm 15,5$ \\
\hline $\mathrm{QR}$ & $1,2 \pm 0,2$ & $1,1 \pm 0,2$ & $1,0 \pm 0,2$ & $1,3 \pm 0,1$ & $1,1 \pm 0,1$ \\
\hline $\mathrm{VO}_{2} \mathrm{NO}_{2} \operatorname{máx}(\mathrm{L} / \mathrm{min})$ & $0,1 \pm 0,1$ & $0,6 \pm 0,1$ & $0,5 \pm 0,2$ & $0,2 \pm 0,04$ & $0,5 \pm 0,1$ \\
\hline $\mathrm{VO}_{2} / \mathrm{VO}_{2}$ máx (\%) & $14,0 \pm 9,1$ & $60,6 \pm 13,9$ & $46,0 \pm 15,1$ & $15,7 \pm 4,4$ & $52,1 \pm 14,5$ \\
\hline Lactato sanguíneo (mmol/L) & $1,2 \pm 0,5$ & $2,7 \pm 1,5$ & - & $1,4 \pm 0,6$ & $2,5 \pm 0,8$ \\
\hline \multicolumn{6}{|l|}{ Ventilatórias } \\
\hline$V_{E}(L / m i n)$ & $7,1 \pm 1,8$ & $19,8 \pm 5,9$ & $15,5 \pm 4,3$ & $7,4 \pm 3,2$ & $18,0 \pm 5,1$ \\
\hline $\mathrm{RV}_{\mathrm{E}}(\%)$ & $81,9 \pm 8,4$ & $52,7 \pm 16,2$ & $61,5 \pm 17,9$ & $84,1 \pm 9,0$ & $54,9 \pm 21,2$ \\
\hline $\mathrm{V}_{\mathrm{E}} / \mathrm{VO}_{2}(\mathrm{~L} / \mathrm{min})$ & $46,9 \pm 24,8$ & $22,4 \pm 2,0$ & $24,3 \pm 5,1$ & $34,7 \pm 6,1$ & $23,9 \pm 5,8$ \\
\hline $\mathrm{V}_{\mathrm{e}} \mathrm{NCO}_{2}(\mathrm{~L} / \mathrm{min})$ & $40,4 \pm 19,4$ & $20,5 \pm 3,3$ & $24,7 \pm 5,1$ & $27,6 \pm 4,3$ & $21,1 \pm 2,9$ \\
\hline $\mathrm{V}_{\mathrm{e}} / \mathrm{VVM}(\mathrm{L} / \mathrm{min})$ & $0,2 \pm 0,1$ & $0,5 \pm 0,2$ & $0,4 \pm 0,2$ & $0,2 \pm 0,1$ & $0,4 \pm 0,2$ \\
\hline $\mathrm{V}_{\mathrm{E}} \mathrm{NVM}(\%)$ & $18,1 \pm 8,4$ & $47,3 \pm 16,2$ & $38,5 \pm 17,9$ & $15,7 \pm 8,5$ & $42,7 \pm 21,2$ \\
\hline \multicolumn{6}{|l|}{ Cardiovasculares } \\
\hline $\mathrm{VO}_{2} / \mathrm{FC}(\mathrm{mL} / \mathrm{bpm})$ & $2,7 \pm 1,9$ & $8,2 \pm 2,0$ & $6,6 \pm 2,0$ & $3,4 \pm 1,1$ & $7,6 \pm 3,1$ \\
\hline RFC (\%) & $46,7 \pm 9,4$ & $27,7 \pm 14,2$ & $32,3 \pm 11,0$ & $51,3 \pm 8,6$ & $27,8 \pm 7,7$ \\
\hline
\end{tabular}

Legenda: Os dados estão apresentados em médias e desvios-padrões; TD6 = teste do degrau de seis minutos; TD2 = isotime de dois minutos do teste do degrau; TSL = teste de sentar e levantar da cadeira de dois minutos; $\mathrm{VO}_{2}=$ consumo de oxigênio; $\mathrm{VCO}_{2}=$ produção de gás carbônico; $\mathrm{RVO}_{2}=$ reserva metabólica; $\mathrm{QR}=$ quociente respiratório; $\mathrm{VO}_{2} / \mathrm{VO}_{2}$ máx $=$ demanda metabólica; $\mathrm{V}_{\mathrm{E}}=$ ventilação minuto; $R V_{E}=$ reserva ventilatória; $V_{\mathrm{E}} / \mathrm{VO}_{2}=$ equivalente ventilatório para o oxigênio; $\mathrm{V}_{\mathrm{E}} / \mathrm{VCO}_{2}=$ equivalente ventilatório para 0 gás carbônico; $\mathrm{V}_{\mathrm{E}} / \mathrm{VVM}$ = demanda ventilatória; $\mathrm{VO}_{2} / \mathrm{FC}=$ pulso de oxigênio; $\mathrm{RFC}=$ reserva de frequência cardíaca. Obs.: valores absolutos e percentuais e resultados estatísticos (análise intertestes).

Fonte: Dados da pesquisa. 
Tabela 3 - Deltas de variação da saturação periférica de oxigênio, frequência cardíaca, frequência respiratória, sensação de dispneia, cansaço/dor nos membros inferiores e pressão arterial diastólica e sistólica e valor da pressão arterial média no TD6, TD2 (isotime) e TSL dos indivíduos com DPOC

\begin{tabular}{lccc}
\hline Variáveis & TD6 & TD2 (isotime) & TSL \\
\hline Ventilatórias & & & \\
\hline$\Delta \mathrm{SpO}_{2}(\%)$ & $3,7 \pm 2,8$ & $2,6 \pm 1,7$ & $3,7 \pm 3,6$ \\
$\Delta \mathrm{FR}(\mathrm{rpm})$ & $4,0 \pm 2,6$ & - & $3,8 \pm 1,0$ \\
\hline Cardiovasculares & & & \\
\hline$\Delta \mathrm{FC}(\mathrm{bpm})$ & $27,9 \pm 19,3$ & $21,8 \pm 11,7$ & $34,6 \pm 16,4^{*}$ \\
$\Delta \mathrm{PAS}(\mathrm{mmHg})$ & $22,5 \pm 24,9$ & - & $19,5 \pm 11,1$ \\
$\Delta \mathrm{PAD}(\mathrm{mmHg})$ & $4,0 \pm 8,8$ & - & $6,4 \pm 5,0$ \\
\hline Sintomas & & & \\
\hline$\Delta$ sensação de dispneia & $1,8 \pm 1,4$ & $0,8 \pm 1,2$ & $0,8 \pm 0,9$ \\
$\Delta$ fadiga de MMII & $1,9 \pm 1,5$ & $0,4 \pm 0,7$ & $1,5 \pm 1,1^{* *}$ \\
\hline
\end{tabular}

Legenda: 0s dados estão apresentados em médias e desvios-padrões; TD6 = teste do degrau de seis minutos; TD2 = isotime de dois minutos do teste do degrau; TSL = teste de sentar e levantar da cadeira de dois minutos; $\mathrm{Sp02}$ = saturação periférica de oxigênio; $F R=$ frequência respiratória; $F C=$ frequência cardíaca; $P A S=$ pressão arterial sistólica; $P A D=$ pressão arterial diastólica; teste t de Student pareado: ${ }^{*}=p<0,04$ (TD2 isotime $\Delta$ TSL pico); ${ }^{* *}=p<0,002$ (TD2 isotime $\Delta$ TSL pico). Obs.: valores absolutos e resultados estatísticos (análise intertestes).

Fonte: Dados da pesquisa

Não houve relatos de sintomas (mal-estar, síncope, dor precordial) espontaneamente pelos indivíduos durante os testes.

Fortes correlações positivas estatisticamente significantes $(\mathrm{p}<0,004)$ foram observadas entre o $\mathrm{VO}_{2}, \Delta \mathrm{FC}$ e os desempenhos físicos no TD2 e TD6 ( $\mathrm{r}$ $=0,86 ; r=0,86$ e $r=0,90$, respectivamente), e entre os desempenhos físicos no TD6 e TSL ( $\mathrm{r}=0,73$; $\mathrm{p}<$ $0,01)$ e entre o TD2 e TSL $(r=0,64 ; p<0,04)$.

\section{Discussão}

Quanto à avaliação das variáveis metabólicas, ventilatórias, cardiovasculares e a dispneia entre os testes submáximos, infere-se que as respostas encontradas foram semelhantes, demonstrando demandas similares, porém constatou-se maior $\Delta \mathrm{FC}$ e $\Delta$ fadiga de MMII no TSL comparado ao TD2, nos indivíduos com DPOC. As fortes correlações positivas encontradas entre o $\mathrm{VO}_{2}, \Delta \mathrm{FC}$ e o número de subidas no degrau no TD2 e TD6, e entre os desempenhos físicos no TD6 e TSL, e no TD2 com o TSL, indicam que esses testes podem avaliar a capacidade funcional desses indivíduos.
Há na literatura poucos estudos que tenham verificado as diferentes respostas metabólicas, ventilatórias, cardiovasculares e perceptuais durante o TD6 comparado a outros testes de campo em indivíduos com DPOC $(7,15,16)$, porém não foram encontrados estudos comparando essas respostas com as do TSL e no TD2.

Quanto às variáveis metabólicas, ventilatórias, cardiovasculares $\left(\mathrm{VO}_{2} / \mathrm{FC}, \mathrm{RFC}, \triangle \mathrm{PAS}\right.$ e $\left.\triangle \mathrm{PAD}\right)$ e $\triangle$ dispneia, não foram observadas diferenças significativas entre os testes, sugerindo estresse metabólico, ventilatório, cardiovascular e dispneia similares em magnitude, em razão da imposição de cargas de trabalho semelhantes, mostrando-se elevadas para esses pacientes (4), podendo ser utilizadas para avaliar a capacidade funcional submáxima dos indivíduos com DPOC.

Os maiores valores de $\triangle \mathrm{FC}$ encontrados para o TSL comparado ao TD2 se traduzem em maior estresse cardiovascular, em razão das exigências metabólicas periféricas e as mudanças posturais envolvidas nesse teste. Entretanto, os testes realizados caracterizam-se como submáximos, o que pode ser melhor demonstrado pelas respostas das variáveis 
cardiovasculares ( $\mathrm{VO}_{2} / \mathrm{FC}$ e $\left.\mathrm{RFC}\right)$, em que atingiram 72,2\%; 67,7\%; e 72\% da FC máxima, no TD6, TD2 e TSL, respectivamente.

Quanto ao $\Delta$ fadiga de MMII, encontraram-se valores significativamente maiores no TSL que no TD2. Tal fato pode ser explicado pela utilização dos membros inferiores simultaneamente, pela maior quantidade de massa muscular ativa na execução da atividade de sentar e levantar da cadeira em relação à atividade de subir e descer o degrau, além da diferença mecânica no movimento, o efeito da ação da gravidade e as mudanças posturais envolvidas, apesar de ambos se caracterizarem como testes estáticos, do teste do degrau envolver a cadeia cinética aberta e fechada e o TSL envolver apenas a cadeia fechada.

De acordo com os dados, observou-se aumento da $\mathrm{V}_{\mathrm{E}} / \mathrm{VVM}$ de $20,4 \%, 29,2 \%$; e $27,0 \%$ no TD2, TD6 e TSL, respectivamente; e da $\mathrm{VO}_{2} / \mathrm{VO}_{2}$ máx de $32,0 \% ; 46,6 \%$; e $36,4 \%$, caracterizando um exercício de intensidade moderada para os indivíduos com DPOC. Assim, sugere-se a utilização do TD2 para indivíduos com DPOC de obstrução grave e muito grave e TD6 para indivíduos com DPOC de obstrução leve a moderada. Pela RFC, constatou-se diminuição de 14,4\%; 19,0\%; e 23,5\% no TD2, TD6 e TSL, respectivamente, indicando que os testes realizados foram submáximos.

Pasqualoto (23) constatou valores significativamente maiores de $\mathrm{VO}_{2}$ no TD6 comparado ao TCP em cicloergômetro e TC6, e também no TCP, que no teste de cadeira (tempo de duração do teste de um minuto); $\mathrm{V}_{\mathrm{E}}$ e a $\mathrm{V}_{\mathrm{E}} / \mathrm{VVM}$ foram significativamente menores no TSL comparado ao TC6, TD6 e TCP; já o TCP apresentou estresse cardiovascular mais intenso que o TC6, TD6 e teste da cadeira; e os valores de $\mathrm{VO}_{2}$ / $\mathrm{FC}$ foram menores no teste da cadeira comparando-se ao TC6 e TD6. Dal Corso et al. (7) encontraram valores metabólicos, ventilatórios, cardiovasculares e perceptuais significativamente maiores no TCP máximo comparado ao TCP submáximo e o TD6.

Corroborando com os achados, Dal Corso et al. (7) não encontraram diferença significante entre o $\Delta \mathrm{SpO}_{2}$ do TD6 e dos testes cardiopulmonares máximo e submáximo realizados em indivíduos com doença pulmonar intersticial de obstrução leve a moderada; concluindo que o TD6 constitui-se em uma alternativa confiável para estimar a capacidade de exercício e o grau de dessaturação. Ao contrário, Ozalevli et al. (8) encontraram valores de $\Delta \mathrm{SpO}_{2}$ significantemente maiores no TC6 que no teste da cadeira realizado nos indivíduos com DPOC, porém nesse último a duração do teste foi de um minuto; e concluíram que o teste da cadeira pode ser usado como método alternativo ao TC6 para avaliar a capacidade de exercício, mas não o grau de dessaturação. Todavia, Montes de Oca et al. (16) constataram que o teste do degrau em indivíduos com DPOC demonstrou melhor relação com o consumo máximo de oxigênio e com a resposta cardíaca do que o TC6, sendo uma alternativa para avaliar a capacidade funcional máxima desses indivíduos, porém o teste do degrau não substitui a medição direta do consumo máximo de oxigênio do teste cardiopulmonar, pelo fato dos autores não terem avaliado as variáveis metabólicas e ventilatórias nesse teste.

Em relação à sensação de dispneia, não houve diferença significante entre os testes, demonstrando demandas perceptuais similares. Tais resultados corroboram com Puente-Maestu et al. (24), os quais observaram que a dispneia não aumentou de forma linear nos testes submáximos em cicloergômetro nas quatro intensidades de trabalho (isto é, 65\%, 75\%, $85 \%$ e $95 \%$ da taxa de trabalho incremental pico), assim como não apresentou diferença significante entre eles; e com Pasqualoto (23), que não observaram diferença entre o TD6 e teste da cadeira. Ao contrário, Dal Corso et al. (7) encontraram maior dispneia e fadiga de MMII no TCP máximo comparado ao submáximo e ao TD6, em indivíduos com doença pulmonar intersticial; e Dreher et al. (25) encontraram que o teste de escada (subir uma escada de 44 degraus com $16 \mathrm{~cm}$ de altura localizada no hospital) resulta em maior hiperinsuflação pulmonar, maior produção de lactato sanguíneo e maior sensação de dispneia do que o TC6, em indivíduos com DPOC de obstrução grave e muito grave, demonstrando demanda metabólica elevada na sua execução; e Ozalevli et al. (8) encontraram valores de $\Delta$ dispneia maiores no TC6 que no teste da cadeira, sendo que nesse último a duração do teste foi de um minuto.

Borel et al. (26) avaliaram a validade do stepper teste (sistema hidráulico, em que o indivíduo realiza o movimento de subida e descida) e concluíram que esse teste é uma opção similar ao TD6 em indivíduos com DPOC e jovens saudáveis, além de avaliar a mesma demanda cardiorrespiratória do TC6, porém o desempenho nesse teste não prediz o desempenho no TC6 e vice-versa. Entretanto, Schnaider e Karsten (15) avaliaram indivíduos DPOC exacerbados hospitalizados por meio do TC6 e TD6 e 
observaram que as respostas cardiorrespiratórias e de percepção de esforço foram equivalentes em ambos os testes e que o desempenho do TD6 prediz o desempenho no TC6, podendo substituí-lo na falta de espaço adequado.

Quanto ao desempenho funcional no TD2 e no TSL, todos os indivíduos executaram os testes de maneira constante e sem interrupção, fato verificado por não observar diferença significativa entre eles; e a relação positiva significativa encontrada sugere que um teste do degrau com o tempo de dois minutos forneceria uma estimativa confiável da capacidade de exercício de indivíduos com DPOC de obstrução grave e muito grave, além de serem suficientes para provocarem alterações metabólicas, ventilatórias, cardiovasculares e perceptuais; resultados esses que corroboram com Dal Corso et al. (7) e Hadeli et al. (27) em estudos realizados com indivíduos com doença pulmonar intersticial, e Casas et al. (1), que constataram que os oito indivíduos com DPOC moderada a grave pertencentes a seu estudo, orientados a subir a escada o mais rápido possível, atingiram aproximadamente $80 \%$ do $\mathrm{VO}_{2}$ pico no final do primeiro minuto do teste de escada; sendo esse teste clinicamente útil para a população estudada.

A diferença significativa encontrada entre o desempenho funcional no TD6 e o TSL atribui-se ao seu tempo de execução. Também foi encontrada forte relação positiva entre o desempenho no TD6 com o TD2 e TSL, sugerindo utilizar o TD6 para estimar a capacidade de endurance da musculatura dos MMII e as limitações funcionais de indivíduos com DPOC de obstrução leve a moderado.

\section{Características dos testes funcionais submáximos}

Comparado ao teste de caminhada de seis minutos, o TD6 envolve maior quantidade de grupos musculares, altos níveis de lactato sanguíneo, maiores requerimentos metabólico e ventilatório, além de apresentar maior impacto na ineficiência da exalação do gás carbônico, ou seja, maior volume de espaço morto/volume corrente em razão das diferenças na postura corporal, volumes pulmonares e/ou hemodinâmica central (28), podendo o mesmo ser dito para o TSL.

A razão pela escolha do tempo de teste de seis minutos para o TD6 foi baseada na validade do teste de caminhada de seis minutos, e de dois minutos para o TSL graças a um estudo piloto, no qual foi observado que os indivíduos não conseguiam realizar repetições de sentar e levantar da cadeira por mais de dois minutos, sendo assim essa justificativa para a escolha do tempo. Não foi escolhido o tempo original de um minuto do TSL (8) por acreditarmos que seria um tempo insuficiente para o indivíduo atingir intensidade máxima dos sintomas e das respostas metabólicas, ventilatórias e cardiovasculares. Outro aspecto importante foi o uso de um degrau simples de $20 \mathrm{~cm}$ de altura, pois degrau com altura inferior poderia fornecer um estresse metabólico insuficiente, isto é, os sujeitos necessitariam subir e descer o degrau o mais rápido possível, em uma velocidade ainda maior do que a executada no presente estudo, o que aumentaria o risco de quedas (7), e um degrau com altura superior poderia ser um instrumento difícil para indivíduos idosos com problemas de joelhos e quadril, apesar de tal fato fazer parte dos critérios de exclusão do estudo.

Implicações clínicas

o TC6, considerado teste clínico de campo, necessita de um corredor com no mínimo 30 metros de comprimento e requer mais de um avaliador para a sua realização (9). Ao contrário, o TD6 requer apenas um degrau simples de $20 \mathrm{~cm}$ de altura, podendo ser portátil e realizado em qualquer local, até mesmo em uma sala de atendimento; e o TSL requer apenas uma cadeira de $46 \mathrm{~cm} \mathrm{(7)} \mathrm{de} \mathrm{altura,} \mathrm{a} \mathrm{qual}$ pode ser encontrada até mesmo no domicílio do indivíduo. Além disso, em ambos a monitorização é simples e o desempenho físico pode ser facilmente registrado, permitindo avaliar a capacidade de exercício em avaliações de rotina; eles apresentam, também, maiores vantagens em termos de redução de custos e aumento da frequência das avaliações funcionais dos indivíduos com DPOC.

Neste estudo, algumas limitações podem ser observadas, como a amostra composta unicamente por indivíduos do sexo masculino; a presença de indivíduos com DPOC de obstrução moderada; o TD6 e TSL vêm sendo utilizados para avaliar o desempenho funcional dos indivíduos com DPOC, mas ainda necessitam serem padronizados; além de não ter sido possível realizar um teste cardiopulmonar máximo e assim compará-lo com os testes executados, a fim de observar o desempenho dos indivíduos diante disso. 


\section{Conclusão}

Os testes realizados e o TD2 foram submáximos e apresentaram respostas metabólicas, ventilatórias, cardiovasculares e dispneia similares nos indivíduos com DPOC, fornecendo informações do grau de comprometimento funcional e do metabolismo muscular, refletindo a disfunção metabólica, ventilatória e/ ou periférica; e o TD2 mostrou-se uma alternativa sensível e confiável para avaliar as limitações funcionais dos indivíduos com DPOC de obstrução grave, apesar de na prática oferecer menor estresse cardiovascular e fadiga muscular comparando-se ao TSL, em razão das exigências metabólicas periféricas.

\section{Referências}

1. Casas A, Vilaro J, Rabinovich R, Mayer A, Barberà JA, Rodriguez-Roisin R, et al. Encouraged 6-min walking test indicates maximum sustainable exercise in COPD patients. Chest. 2005;128(1):55-61.

2. Ferrazza AM, Martolini D, Valli G, Palange P. Cardiopulmonary exercise testing in the functional and prognostic evaluation of patients with pulmonary diseases. Respiration. 2009;77(1):3-17.

3. Leung ASY, Chan KK, Sykes K, Chan KS. Reliability, validity, and responsiveness of a 2-min walk test to assess exercise capacity of COPD patients. Chest. 2006;130(1):119-25.

4. Neder JR, Nery LE. Fisiologia clínica do exercício. São Paulo: Artes Médicas; 2003.

5. Cataneo DC, Cataneo AJM. Acurácia do teste de escada utilizando o consumo máximo de oxigênio como padrão-ouro. J Bras Pneumol. 2007;33(2):128-33.

6. Dourado VZ, Tanni SE, Vale AS, Faganello MM, Sanchez FF, Godoy I. Manifestações sistêmicas na doença pulmonar obstrutiva crônica. J Bras Pneumol. 2006;32(2):161-71.

7. Dal Corso S, Duarte SR, Neder JA, Malaguti C, Fuccio MB, Castro Pereira CA, et al. A step test to assess exercise-related oxygen desaturation in interstitial lung disease. Eur Respir J. 2007;29(2):330-6.

8. Ozalevli S, Ozden A, Itil O, Akkoclu A. Comparison of the sit-to-stand test with 6 min walk test in patients with chronic obstructive pulmonary disease. Respir Med. 2007;101(2):286-93.
9. American Thoracic Society. ATS Statement: guidelines for the six-minute walk test. Am J Respir Crit Care Med. 2002;166(1):111-7.

10. Celli BR, Cote CG, Marin JM, Casanova C, Montes de Oca M, Mendez RA, et al. The body mass index, Airflow obstruction, dyspnea, and exercise capacity index in chronic obstructive pulmonary disease. $\mathrm{N}$ Engl J Med. 2004;350(10):1005-12.

11. Cahalin LP, Mathier MA, Semigran MJ, Dec GW, Disalvo TG. The six-minute walk test predicts peak oxygen uptake and survival in patients with advanced heart failure. Chest. 1996;110(2):325-32.

12. Pitta F, Troosters T, Spruit MA, Decramer PM, Gosselink R. Characteristics of physical activities in daily life in chronic obstructive pulmonary disease. Am J Respir Crit Care Med. 2005;171(9):972-7.

13. Balfour-Lynn IM, Prasad SA, Laverty A, Whitehead BF, Dinwiddie R. A step in the right direction: assessing exercise tolerance in cystic fibrosis. Pediatr Pulmonol. 1998;25(4):278-84.

14. Swinburn CR, Wakefield JM, Jones PW. Performance, ventilation, and oxygen consumption in three different types of exercise test in patients with chronic obstructive lung disease. Thorax. 1985;40(8):581-6.

15. Schnaider J, Karsten M. Testes de tolerância ao exercício em programa de fisioterapia hospitalar após exacerbação da doença pulmonar obstrutiva crônica. Fisioter Mov. 2006;19(4):119-26.

16. Montes de Oca M, Ortega Balza M, Lezama J, López JM. Chronic obstructive pulmonary disease: evaluation of exercise tolerance using three different exercise tests. Arch Bronconeumol. 2001;37(2):69-74.

17. Rabe KF, Hurd S, Anzueto A, Barnes PJ, Buist SA, Calverley P, et al. Global strategy for the diagnosis, management, and prevention of chronic obstructive pulmonary disease: GOLD Executive Summary. Am J Respir Crit Care Med. 2007;176(6):532-55.

18. Bertolucci PH, Brucki SM, Campacci SR, Juliano Y. The mini-mental state examination in a general population: impact of educational status. Arq Neuropsiquiatr. 1994;52(1):1-7.

19. Pereira CAC, Sato T. Limitação ao fluxo aéreo e capacidade vital reduzida: distúrbio ventilatório obstrutivo ou combinado? J Bras Pneumol. 1991;17:59-68. 
20. Miller MR, Hankinson J, Brusasco V, Burgos F, Casaburi R, Coates A, et al. ATS/ERS task force: standardisation of lung function testing. Eur Respir J. 2010;36(6):1496-8.

21. Casanova C, Cote C, Marin JM, Pinto-Plata V, Torres JP, Aguirre-Jaíme A, et al. Distance and oxygen desaturation during the 6-min walk test as predictors of long-term mortality in patients with COPD. Chest. 2008;134(4):746-52.

22. Borg GA. Psychophysical bases of perceived exertion. Med Sci Sports Exerc. 1982;14(5):377-81.

23. Pasqualoto AS. Comparação das respostas fisiológicas no teste de exercício cardiopulmonar e em três testes de exercício submáximo em pacientes com doença pulmonar obstrutiva crônica [tese]. Porto Alegre: Universidade Federal do Rio Grande do Sul; 2009.

24. Puente-Maestu L, Pedro JG, Martínez-Abad Y, Oña JMR, Llorente D, Cubillo JM. Dyspnea, ventilatory pattern, and changes in dynamic hyperinflation related to the intensity of constant work rate exercise in COPD. Chest. 2005;128(2):651-6.

25. Dreher M, Walterspacher S, Sonntag F, Prettin S, Kabitz HJ, Windisch W. Exercise in severe COPD: is walking different from stair-climbing? Respir Med. 2008;102(6):912-8.
26. Borel B, Fabre C, Saison S, Bart F, Grosbois JM. An original field evalution test for chronic obstructive pulmonary disease population: the six-minute stepper test. Clin Rehabil. 2010;24(1):82-93.

27. Hadeli KO, Siegel EM, Sherril DL, Beck KC, Enright PL. Predictors of oxygen desaturation during submaximal exercise in 8,000 patients. Chest. 2001; 120(1):88-92.

28. Palange P, Forte S, Onorati P, Manfredi F, Serra P, Carlone S. Ventilatory and metabolic adaptations to walking and cycling in patients with COPD. J Appl Physiol. 2000;88(5):1715-20.

Recebido: $16 / 11 / 2010$

Received: $11 / 16 / 2010$

Aprovado: 20/05/2011

Approved: 05/20/2011 